\title{
Participação em programas para a terceira idade: impacto sobre a cognição, humor e satisfação com a vida
}

\author{
Participation in programs for seniors: \\ impact on cognition, mood \\ and life satisfaction
}

\author{
Mônica Sanches YASSUDA \\ Henrique Salmazo da SILVA ${ }^{2}$
}

\begin{abstract}
Resumo
Este estudo teve como objetivo investigar a relação entre a participação em programas da terceira idade e os possíveis benefícios para o desempenho cognitivo, humor e satisfação com a vida. Vinte e nove idosos que tinham iniciado participação em quatro centros de convivência foram submetidos a duas avaliações: no início da participação e após seis meses de atividades no grupo. As avaliações foram compostas pelas escalas: Mini-exame do Estado Mental, Lista de Palavras do Consortium to Establish a Registry of Alzheimer Disease, Teste de Fluência Verbal Categoria Animais, Escala de Depressão Geriátrica e Escala de Satisfação com a Vida. Os resultados indicaram que a participação em programas sociais parece gerar benefícios: aumento significativo no resgate das palavras do Consortium to Establish a Registry of Alzheimer Disease e aumento, que se aproximou da significância estatística, da fluência verbal e satisfação com a vida em relação ao envolvimento social. Os efeitos do engajamento da população idosa nesses programas devem ser investigados.
\end{abstract}

Unitermos: Envelhecimento. Grupos de convivência. Idosos. Satisfação pessoal.

\begin{abstract}
The purpose of this research was to investigate the relationship between participation in social activities in senior citizens centers and the potential benefits for cognitive performance, mood and life satisfaction. Twenty-nine seniors, who had recently become members of four senior citizens centers, each completed two evaluations: one prior to participation and the other after six months of group activities. Assessment protocol included the following scales: Mini Mental Status Examination, Word List of the Consortium to Establish a Registry for Alzheimer's Disease, Verbal Fluency Test in the Animals Category, Geriatric Depression Scale, and the Life Satisfaction Scale. Results indicated that participation in social programs can generate benefits: a significant increase was detected in word recall and also an improvement, approaching statistical significance, in word fluency and life satisfaction in terms of social involvement. The impact of the involvement of the elderly in these programs needs to be investigated.
\end{abstract}

Uniterms: Aging. Senior citizens centers. Elderly. Personal satisfaction.

$\boldsymbol{\nabla \nabla \nabla v}$

1 Universidade de São Paulo, Escola de Artes, Ciências e Humanidades, Curso de Gerontologia. Av. Arlindo Bettio, 1000, Ermelino Matarazzo, 03828-000, São Paulo, SP, Brasil. Correspondência para/Correspondence to: M.S. YASSUDA. E-mail: <yassuda@usp.br>.

2 Universidade de São Paulo, Faculdade de Saúde Pública, Departamento de Epidemiologia. São Paulo, SP, Brasil. 
A criação de novos espaços e novas formas para envelhecer é uma realidade relativamente nova no contexto brasileiro. No início das décadas de 1960 e 1970, alguns teóricos e profissionais comprometidos com o processo de envelhecimento começaram a se questionar sobre a possibilidade de implementar programas de caráter sociocultural e lúdico para a população idosa. Tais programas propiciariam experiências enriquecedoras como forma de ocupação do tempo livre, redução do isolamento social, inserção do idoso na comunidade e ainda estruturação de grupos efetivos de apoio (Cachioni \& Palma, 2006; Ferrigno, Leite \& Abigalil, 2006; Salgado, 1982; Veras \& Caldas, 2004).

Instituições como o Serviço Social do Comércio (SESC) e, posteriormente, as Universidades Abertas para a Terceira Idade foram pioneiras na elaboração das primeiras iniciativas no Brasil, possibilitando que experiências desenvolvidas em países como a França pudessem ser transpostas para a realidade brasileira. Após cerca de 40 anos de atuação, as atividades desenvolvidas pelos centros de convivência, universidades abertas e escolas abertas passaram a ser difundidas entre os profissionais gerontólogos, pelos veículos de comunicação e pelas próprias pessoas que envelhecem como maneiras de alcançar a velhice bem-sucedida (Debert, 1999).

Apesar de a sociedade considerar que programas voltados à população idosa são justificáveis e relevantes, pesquisas científicas revelam achados controversos. Estudos recentes que investigaram o desempenho cognitivo em idosos vivendo na comunidade sugerem forte associação entre o engajamento em atividades mentais estimulantes e o desempenho cognitivo (Hultsch, Hertzog, Small, McDonald-Miszczak \& Dixon, 1992; Hultsch, Hertzog, Small, \& Dixon, 1999; Newson \& Kemps, 2005; Lövdén, Ghisletta \& Linderberger, 2005). No estudo de Lövdén et al. (2005), os autores acompanharam idosos vinculados ao Berlin Aging Study (BASE) e verificaram que as atividades sociais estavam associadas ao aumento na velocidade de processamento das informações. Em contrapartida, os resultados de Aarsten, Smits, van Tillrug, Knipscheer e Dag (2002), MacKinnon, Christensen, Hofer, Kater e John (2003) e Salthouse, Berish e Miles (2002) não encontraram associações significativas entre a realização de atividades e a mudança no desempenho cognitivo na velhice. No estudo de Mackinnon et al. (2003), foi observado que a saúde é um grande preditivo de desempenho cognitivo na velhice e que um modelo eficaz para a investigação da causalidade e direcionalidade dos efeitos da atividade sobre a cognição no final da vida deve incluir o controle de variáveis de saúde, nível de atividade e estilo de vida.

Quanto ao impacto da participação nos programas sociais sobre variáveis psicossocais, estudos encontraram associação entre a não participação em atividades comunitárias e maiores índices de sintomas depressivos, sugerindo que a filiação em atividades sociais pode gerar a consolidação de redes de suporte social e aumentar a satisfação com a vida (Antonucci, Fuhrer \& Dartigues, 1997; Antonucci, 2001; Blazer, 2005; Gazzale, Lima, Tavares \& Hallal, 2004). Harlow e Cantor (1996) indicaram que a participação em vida social e em serviços comunitários é preditiva de satisfação com a vida, melhor controle da saúde, presença de maior suporte social e bem-estar subjetivo na velhice.

Em geral, a participação em programas e serviços comunitários está associada à redução dos indicadores de stress, aumento dos sentimentos e emoções positivas, controle da saúde, ampliação das ofertas de suporte social e exercício da habilidade de resolução de problemas (Borges, 2006; Cachioni \& Palmas, 2006; Debert, 1999; Deps, 1993; Ferrigno et al., 2006; Harlow \& Cantor, 1996; Lopes, 2006; Mercadante, 2002; Neri, 1993; Neri, 2003; Rocha, Gomes \& Lima Filho, 2002). Nos estudos de Irigaray e Schneider (2008) e Loures e Gomes (2006), promovidos no contexto dos programas Universidade Aberta para a Terceira Idade, foi verificada redução significativa dos sintomas depressivos após participação nas atividades lúdicas e socioeducativas. Irigaray e Schneider (2008), ao avaliar 103 participantes idosas no intervalo de um ano, descobriram que, além da redução dos sintomas depressivos, houve aumento da qualidade de vida das participantes em relação aos domínios físico, psicológico e social. Assim, programas oferecidos aos idosos residentes na comunidade podem ter um papel relevante em medidas de prevenção na área de saúde mental (Gatz \& Smyer, 2001; Ramos, 2003).

Profissionais e pesquisadores gerontólogos entendem que a participação em programas para a terceira idade gera benefícios psicológicos, sociais e de saúde. Poucos estudos, entretanto, tentaram quantificar o impacto da participação nessas atividades. O presente 
estudo teve como objetivo investigar se a participação em programas voltados para a terceira idade gera benefícios para a cognição, humor e satisfação com a vida.

\section{Método}

\section{Participantes}

Foram entrevistados 29 idosos que haviam se inscrito em atividades sociais e lúdicas oferecidas em quatro centros de convivência em Ermelino Matarazzo, São Paulo. Dos participantes, 70\% eram do gênero feminino e 30\%, do masculino.

Os participantes foram reavaliados após seis meses de participação nas atividades. Na avaliação de segmento, 12 participantes deixaram de frequentar os programas e somente 17 participantes foram reentrevistados. Os principais motivos para a saída desses participantes foram: mudança para outras cidades ou retorno à cidade natal, engajamento em atividades remuneradas ou trabalho informal, tratamento médico e distância entre o local onde ocorriam as atividades e a residência.

O critérios de seleção dos participantes foi: ter idade igual ou superior a 50 anos; residir na comunidade de Ermelino Matarazzo; não apresentar sinais sugestivos de demência nas avaliações do estudo; e ter iniciado as atividades nos grupos de convivência pesquisados entre abril e junho de 2005. Os 29 idosos inicialmente avaliados preencheram todos os critérios.

A média etária dos participantes foi de 66,84 anos, Desvio-Padrão (DP) de 8,24, variando entre 52 a 90 anos; $60,5 \%$ da amostra apresentavam idade igual ou inferior a 70 anos, o que indica que os participantes eram relativamente jovens. Quanto ao nível de escolaridade, a média de anos de estudo foi de 3,16 anos (DP=2,61) e 75,9\% dos participantes sabiam ler e escrever, com grau de escolaridade entre zero e quatro anos. Quanto ao estado civil e etnia, 52,0\% dos participantes eram viúvos e 48,0\% referiram pertencer à etnia branca.

Em relação à renda, 37,9\% dos pesquisados apresentavam rendimento mensal inferior a um salário-mínimo ao mês, e 42,0\% recebiam entre um e dois salários. A maioria dos participantes - 89,7\% - referiram ser aposentados e apenas 10,3\% obtinham rendimentos provenientes de trabalho informal. Quanto à região de origem, 11 idosos eram provenientes do meio rural e 18 do meio urbano.

O único fator que se diferenciou entre o grupo de participantes que permaneceu nos programas e os 12 participantes que saíram foi a variável idade. A média etária dos participantes que saíram foi de 59,5 anos $(\mathrm{DP}=5,38)$, enquanto a média etária dos participantes que permaneceram nos programas foi de 70,2 anos $(\mathrm{DP}=7,06)$. Não se detectou diferença significativa nas outras variáveis sociodemográficas entre os dois grupos.

Dos 12 equipamentos sociais disponíveis na região pesquisada, quatro centros de convivência para idosos foram selecionados, com base nos seguintes critérios: 1) desenvolver atividades em oficinas de artesanato (tricô, crochê e ponto cruz), dança, oficinas socioeducativas, oficinas de teatro, acompanhamento psicológico, jogos (baralho, dominó e bocha), programas de alfabetização, bingo, bazares, excursões e passeios, e aulas sobre saúde e envelhecimento; 2) oferecer atividades com periodicidade de, pelo menos, duas vezes por semana; 3) aprovar a realização da pesquisa e conceder uma sala para que as entrevistas fossem realizadas.

Para preservar o nome das entidades, as instituições foram caracterizadas como instituição A, B, C e D; sendo 15 participantes provenientes da instituição $A$, quatro da instituição $B$, quatro da C e seis da D.

\section{Procedimentos}

Nesta pesquisa, foram aplicados testes de rastreio cognitivo e escalas que avaliam o humor e a satisfação com a vida referenciada aos domínios saúde, bem-estar físico, engajamento social e saúde mental, instrumentos já validados para a população brasileira. Os testes utilizados foram o Miniexame do Estado Mental (MEEM) (Bertolucci, Bruchy, Canipacci \& Juliano, 1994; Brucki, Nitrini, Carameli, Bertolucci \& Okamoto, 2003; M.F. Folstein, S.E. Folstein \& McHugh, 1975), a Lista de Palavras do Consortium to Establish a Registry of Alzheimer Disease (CERAD) (Bertolucci, Okamoto, Brucki, Siveiro, Toniolo Neto \& Ramos, 2001), Fluência Verbal Categoria Animais (Brucki \& Rocha, 2004), a Escala de Depressão Geriátrica (O. Almeida \&S.A. Almeida, 1999) e a Escala de Satisfação com a Vida (Neri, 1998). Esses instrumentos 
encontram-se descritos a seguir e possuem níveis satisfatórios de sensibilidade e especificidade, isto é, boa capacidade para distinguir entre os possíveis casos e não casos de demência ou de sintomas depressivos, à exceção da Escala de Satisfação com a Vida, que não é um instrumento de rastreio.

O MEEM tem por objetivo rastrear sinais de declínio cognitivo através da avaliação de funções como memória, atenção, linguagem e praxia através de 20 questões. O instrumento foi criado por M. F. Folstein, S.E. Folstein e McHugh (1975) e teve sua aplicabilidade testada na população idosa brasileira em diversos estudos (Bertolucci et al., 1994; Brucki et al., 2003). Neste estudo, utilizou-se a pontuação total que varia de zero a 30 pontos. Como ponto de corte, que indicaria a exclusão de um determinado participante, foi utilizado um desvio-padrão abaixo das medianas apresentadas no estudo conduzido por Brucki et al. (2003) em idosos com diferentes níveis de escolaridade.

A lista de palavras da bateria CERAD é uma prova de memória episódica que avalia a memorização de dez palavras apresentadas três vezes consecutivamente. Utilizou-se a soma das palavras recordadas nos três resgates, variando de zero a 30 pontos, e o resgate tardio após dez minutos, que variou entre zero e 10 pontos.

No instrumento de Fluência Verbal (FV), foi solicitado ao idoso que falasse em um minuto todos os animais que conseguisse recordar (Brucki \& Rocha, 2004). A prova de Fluência Verbal avalia as funções executivas e a linguagem, podendo ser utilizada para avaliar também a memória semântica.

A Escala de Depressão Geriátrica (EDG), validada para a população idosa brasileira por O. Almeida e S.A. Almeida (1999), tem 15 itens, foi adaptada da versão proposta por Yesavage et al. (1983) e avalia a frequência de sintomas depressivos na população idosa. 0 ponto de corte utilizado no presente estudo foi de seis pontos.

A Escala de Satisfação com a Vida (ESV) é composta por 12 questões e avalia o quanto o idoso está satisfeito com sua situação no domínio saúde física, mental, envolvimento social e satisfação global (Neri, 1998). Para avaliar a satisfação em cada domínio, a ESV apresenta questões sobre a satisfação do idoso em relação ao seu estado atual, em comparação com seu estado de dez anos atrás e em comparação com as pessoas da sua idade. As respostas são indicadas em escalas Likert, variando entre 1 e 5 pontos: muito pouco satisfeito (1), pouco satisfeito (2), mais ou menos satisfeito (3), muito satisfeito (4), muitíssimo satisfeito (5). O cálculo das pontuações para os domínios saúde física, mental, envolvimento social e satisfação global foi realizado por meio do agrupamento dos resultados das questões referentes à satisfação atual, satisfação em relação a dez anos atrás e em comparação com as pessoas da mesma faixa etária.

O idoso foi convidado a participar da pesquisa ao se matricular nas instituições. Após o preenchimento do consentimento livre e esclarecido, elaborado com base na Resolução 196/96, o idoso completou a avaliação realizada individualmente em aproximadamente 60 minutos. O protocolo de pesquisa e o estudo fazem parte de um programa amplo de pesquisas sobre envelhecimento cognitivo, que foi apreciado e aprovado pelo Comitê de Ética do Instituto de Psicologia da Universidade de São Paulo (IP/USP). Protocolo n०005/08-CEPH-IP/07/02/2008

A variável independente no presente estudo foi o momento de avaliação (avaliação 1 x avaliação 2). As variáveis dependentes foram as medidas de desempenho cognitivo, a saber: status cognitivo global (MEEM), memória episódica (CERAD), funções executivas (Fluência Verbal), sintomas depressivos (EDG) e variáveis de satisfação com a vida referenciada a domínios (ESV).

De forma geral, a análise de dados buscou avaliar se houve diferença nas variáveis dependentes nos dois momentos de avaliação (no início da participação e após seis meses de frequência). O teste de KolmogorovSmirnov foi utilizado para verificar se as variáveis seguiam distribuição normal. Os resultados indicaram que todas as variáveis seguiram distribuição normal, assim optou-se pelo uso de testes paramétricos. O teste t de Student foi utilizado para comparar o desempenho cognitivo dos idosos que deixaram os programas com os idosos que permaneceram. Por meio de ANOVA para medidas repetidas, avaliou-se o efeito do fator Tempo (Avaliação 1 x Avaliação 2). O valor de significância escolhido para essas análises foi de $p<0,05$.

\section{Resultados}

A Tabela 1 apresenta as médias e desvios-padrão para as variáveis cognitivas, de humor e de satisfação 
com a vida para o grupo de participantes que permaneceu nas instituições até a reavaliação após seis meses e para a amostra que saiu dos programas. Comparações estatísticas realizadas através do teste $t$ de Student sugeriram que os dois grupos eram equivalentes quanto ao desempenho cognitivo, ao número de sintomas depressivos e à satisfação com a vida.

Para analisar se houve mudança significativa entre a primeira e a segunda avaliação, as variáveis cognitivas (MEEM, palavras do CERAD e fluência verbal), de humor (EDG) e de satisfação com a vida foram submetidas a ANOVA para medidas repetidas (Fator Tempo: Avaliação 1 x Avaliação 2). Para algumas variáveis, não foram detectadas alterações significativas entre os dois momentos de avaliação, como, por exemplo, para o $\operatorname{MEEM}(F=0,074, p=0,79)$, palavras do CERAD resgate tardio $(F=0,65, p=0,43)$ e a EDG $(F=2,13, p=0,15)($ Tabela 2).

Para o CERAD total (soma dos três resgates imediatos), houve diferença significativa entre os dois momentos ( $F=20,37, p=0,000$ ), com melhor desempenho na segunda avaliação. Para fluência verbal, a diferença entre os dois momentos aproximou-se da significância estatística ( $F=3,31, p=0,088$ ), com melhor desempenho na segunda avaliação. Para as questões sobre satisfação com a vida, não foram detectadas diferenças entre os dois momentos para satisfação com a saúde ( $F=0,063$, $p=0,81)$, satisfação com a saúde física $(F=0,92, p=0,35)$ e satisfação com a capacidade mental ( $F=0,15, p=0,70)$. Aproximou-se da significância estatística a diferença para a satisfação com o envolvimento social ( $F=4,18$, $p=0,058)$, com maior satisfação na segunda avaliação.

Tabela 1.Média e desvio-padrão do desempenho do grupo de participantes que permaneceu nos programas e do grupo de participantes que saíram. São Paulo (SP), 2005.

\begin{tabular}{|c|c|c|c|c|}
\hline \multirow{2}{*}{ Teste } & \multicolumn{2}{|c|}{ Idosos que permaneceram nos programas $(n=17)$} & \multicolumn{2}{|c|}{ Idosos que saíram dos programas $(n=12)$} \\
\hline & $\mathrm{M}$ & $\mathrm{DP}$ & $\mathrm{M}$ & $\mathrm{DP}$ \\
\hline MEEM & 23,60 & 4,55 & 23,41 & 4,40 \\
\hline CERAD 3 resgates & 16,40 & 6,75 & 16,29 & 5,01 \\
\hline EDG & 4,20 & 3,68 & 3,35 & 1,54 \\
\hline ESV1 - saúde & 2,70 & 0,95 & 3,29 & 0,85 \\
\hline ESV4 - capacidade física & 3,40 & 1,07 & 3,71 & 1,16 \\
\hline ESV7 - capacidade mental & 3,20 & 0,79 & 3,65 & 0,93 \\
\hline ESV10 - envolvimento social & 3,67 & 0,92 & 3,65 & 0,79 \\
\hline CERAD resgate tardio & 6,00 & 2,54 & 5,24 & 2,73 \\
\hline Fluência verbal & 12,59 & 2,00 & 11,06 & 3,67 \\
\hline
\end{tabular}

MEEM: mini-exame do estado mental; EDG: escala de depressão geriátrica; ESV: escala de satisfação com a vida; M: média; DP: desvio-padrão.

Tabela 2. Média e desvio-padrão do desempenho no início da participação e após seis meses de frequência, em médias e desvios padrão ( $n=17)$. São Paulo (SP), 2005.

\begin{tabular}{|c|c|c|c|c|c|c|}
\hline \multirow{2}{*}{ Teste } & \multicolumn{2}{|c|}{$1^{\text {a }}$ avaliação } & \multicolumn{2}{|c|}{$2^{a}$ avaliação } & \multirow{2}{*}{\multicolumn{2}{|c|}{ Significância Estatística ANOVA }} \\
\hline & M & DP & M & DP & & \\
\hline MEEM & 23,41 & 4,40 & 23,59 & 3,34 & $F=0,074$ & $p=0,790$ \\
\hline CERAD 3 resgates & 16,26 & 5,01 & 19,12 & 4,86 & $F=20,37$ & $p=0,000^{* *}$ \\
\hline EDG & 3,35 & 1,54 & 2,76 & 1,15 & $F=2,130$ & $p=0,150$ \\
\hline ESV1 - saúde & 3,29 & 0,85 & 3,24 & 0,97 & $F=0,063$ & $p=0,810$ \\
\hline ESV4 - capacidade física & 3,71 & 1,16 & 3,41 & 1,12 & $F=0,920$ & $p=0,350$ \\
\hline ESV7 - capacidade mental & 3,65 & 0,93 & 3,76 & 0,97 & $F=0,150$ & $p=0,150$ \\
\hline ESV10 - envolvimento social & 3,65 & 0,79 & 4,18 & 0,81 & $F=4,180$ & $p=0,058^{*}$ \\
\hline CERAD resgate tardio & 5,24 & 2,73 & 5,74 & 2,83 & $F=0,650$ & $p=0,430$ \\
\hline Fluência verbal & 11,06 & 3,67 & 12,35 & 4,21 & $F=3,310$ & $p=0,088^{*}$ \\
\hline
\end{tabular}

${ }^{*}$ Valor próximo ou igual a $p \leq 0,05 ;{ }^{* *}$ Valor igual a $p \leq 0,005$

MEEM: mini-exame do estado mental; EDG: escala de depressão geriátrica; ESV: escala de satisfação com a vida; M: média; DP: desvio-padrão. 


\section{Discussão}

O presente estudo compreendeu uma tentativa de mapear os benefícios cognitivos e associados ao humor è̀ satisfação com a vida em idosos que se afiliam a centros de convivência e programas da terceira idade. Os dados apresentados sugerem que a participação nas atividades oferecidas pelos programas propiciou alteração no desempenho cognitivo e maior satisfação em relação ao envolvimento social. Houve aumento estatisticamente significativo no pós-teste para a tarefa de memória episódica e ganhos marginalmente significativos para o desempenho no teste de fluência verbal e para a satisfação com a vida referenciada ao domínio envolvimento social.

Os resultados obtidos estão em consonância com alguns achados disponíveis na literatura, sugerindo que a realização de atividades parece favorecer o desempenho cognitivo na velhice (Hultsch et al., 1992; Hultsch et al.,1999; Newson \& Kemps, 2005; Lövden, Ghisletta \& Linderberger, 2005). Esses estudos revelam que possuir um estilo de vida saudável e participar de atividades sociais e lúdicas aumentam as oportunidades de atingir níveis mais elevados de desempenho cognitivo durante a velhice.

Em relação à satisfação com a vida, o aumento marginalmente significativo para a satisfação com a vida em relação ao envolvimento social confirma os dados de Deps (1993), Harlowe Cantor (1996), Mercadante (2002) e Petrini (1997), que indicam que a participação em grupos comunitários contribui para o maior oferecimento de suporte social. A estabilidade dos outros valores referentes à satisfação com a vida quanto aos domínios saúde, capacidade mental e capacidade física, no entanto, podem indicar que os programas contribuem para a estabilidade desses parâmetros em um período de curto prazo, ou que os programas não contribuem para o aumento desses domínios. Para testar essas hipóteses, deve-se construir análises longitudinais ou estudos de seguimento que permitiriam acompanhar os participantes por um intervalo maior de tempo.

Diferentemente dos estudos de Irigaray e Schneider (2008) e Loures e Gomes (2006), o índice de sintomas depressivos não diminuiu na avaliação de seguimento do presente estudo. Uma das razões para os sintomas depressivos permanecerem estáveis na

212 reavaliação pode ser o intervalo de avaliação, que foi reduzido. Poderia se esperar que, por intensificar os contatos sociais, os idosos apresentassem diminuição nos sintomas depressivos, assim como evidenciam Antonucci, Fuhrer e Dartigues (1997), Antonucci (2001) e Blazer (2005) em estudos desenvolvidos com idosos saudáveis e não institucionalizados.

Além dos fatores supracitados, vale mencionar que os resultados apresentados têm inúmeras limitações e devem ser interpretados com cautela. Por tratar-se de estudo exploratório sobre a temática, contou com a investigação de um grupo reduzido de participantes (29 na primeira avaliação e 17 na segunda), de instituições, e de uma amostra que se apresenta com um perfil socioeconômico específico, com baixa escolaridade e rendimento mensal muito próximo a um salário mínimo. Os resultados também não podem ser amplamente generalizados por não incluírem comparação com um grupo-controle, que não participou dos programas.

Em síntese, conclui-se que os centros de convivência e os programas da terceira idade parecem contribuir para o status cognitivo e para a satisfação com a vida dos participantes. Verifica-se que os aspectos cognitivos e psicossociais do envelhecimento revelam plasticidade e podem sofrer a modulação de intervenções socioculturais, como as desenvolvidas pelos centros de convivência. Ações e políticas públicas deveriam ser destinadas para a população idosa residente em regiões periféricas de grandes centros urbanos (Maia, Durante \& Ramos, 2004; Ramos, 2003; Scafuzca et al., 2002), pois essas podem ser aliadas à atenção primária em saúde mental e colaborar para a qualidade de vida dos idosos que vivem nessas regiões.

Futuros estudos devem incluir grupo-controle para verificação dos efeitos de retestagem. Assim, deve-se avaliar se os participantes do estudo não tiveram melhor desempenho na segunda avaliação devido à experiência prévia com os testes de rastreio cognitivo na primeira avaliação. Vale destacar, nesse sentido, que os estudos que examinam o desempenho cognitivo dos idosos e os fatores que contribuem para preservação do status cognitivo são realizados, em geral, por períodos relativamente longos, em intervalos de três (Hultsch et al., 1992), cinco (Albert et al., 1995) e até dezesseis anos (Zelinski \& Stewart, 1998). Assim, outros estudos devem incluir avaliações de seguimento após intervalos mais longos de participação nas atividades. 


\section{Referências}

Aarsten, M. J., Smits, C. H., van Tilburg, T., Knipscheer, K. C. P., \& Deeg, J. H. (2002). Activity in older aldults: cause or consequence of cognitive functioning? A longitudinal study on everyday activities and cognitive performance in olders adults. The Journals of Gerontology, $57 B$ (2), 153-162.

Albert, M. S., Jones, K., Savage, C. R., Berkman, L., Seeman, T., Blazer, D., et al. (1995) Predictors of cognitive change in older persons: MacArthur studies of successfull aging. Psychology and Aging, 10 (4), 578-589.

Almeida, O., \& Almeida, S. A. (1999). Reability of the Brasilian version of the geriatric scale (GDS) short form. Arquivos de Neuropsiquiatria, 57 (B), 421-426.

Antonucci, T. C. (2001). Social relations: an examination of social networks, social support, and sense of control. In J. E. Birren \& K. W. Schaie (Eds.), Handbook of the psychology of aging ( $5^{\text {th }}$ ed., pp.427-448). San Diego, CA: Academic Press.

Antonucci, T. C., Fuhrer, R., \& Dartigues, J-F. (1997). Social relations and depressive symptomatology in a sample of community: dwelling french older adults. Psychology and Aging, 12 (1), 189-195.

Bertolucci, P. H. C., Okamoto, I. H., Brucki, S. M. D., Siveiro, M. O., Toniolo Neto, J., \& Ramos, L. R. (2001). Applicability of the CERAD neuropsychological battery of the Brazilian Elderly. Arquivos de Neuropsiquiatria, 59 (3A), 532-536.

Bertolucci, P. H. C.; Brucky, S. M. D.; Campacci, S. R., \& Juliano, I. (1994). O Mini exame do estado mental em uma população geral: impacto da escolaridade. Arquivos de Neuro-Psiquiatria, 52,1-7.

Blazer, D. (2005). Depression and social support in late life: a clear but not obvious relationship. Aging \& Mental Health, 9 (6), 497-499

Borges, L. C. (2006). Os grupos de convivência na terceira idade: suporte social e afetivo. In D. V. S. Falcão \& C. M. S. B. Dias (Orgs.), Maturidadeevelhice:pesquisas eintervenções psicológicas, (Vol.1, pp.151-165). São Paulo: Casa do Psicólogo.

Brucki, S. M. D., Nitrini, R., Carameli, P., Bertolucci, P. H. F., \& Okamoto, I. H. (2003). Sugestões para o uso do miniexame do estado mental no Brasil. Arquivos de NeuroPsiquiatria, 61 (3B), 777-781.

Brucki, S. M. D., \& Rocha, M. S. G. (2004). Category fluency test: effectes of age, gender and education on total scores, clustering and switching in Brazilian Portuguese-speaking subjects. Brazilian Journal of Medical and Biological Research, 37 (12), 1771-1777.

Cachioni, M., \& Palma, L. S. (2006). Educação permanente: perspectiva para o trabalho educacional com o adulto maduro e idoso. In E. L. Freitas, L. Py, F. A. X. Cançado, J. Doll, M. L. Gorzoni \& S. M. Rocha (Eds.), Tratado de geriatria e gerontologia (2a ed., pp.1456-1465). Rio de Janeiro: Guanabara Koogan.

Debert, G. G. (1999). A reinvenção da velhice: sociabilização e processos de reprivatização do envelhecimento. São Paulo: Fapesp.
Deps, V. L. (1993). Atividade e bem-estar psicológico na maturidade. In A. L. Neri (Org.), Qualidade de vida e idade madura (pp.57-82). Campinas: Papirus.

Ferrigno, J. C., Leite, M. L. C. B., \& Abigalil, A. (2006). Centros e grupos de convivência de idosos: da conquista ao lazer ao direito do exercício da cidadania. In E. L. Freitas, L. Py, F. A. X. Cançado, J. Doll, M. L. Gorzoni \& S. M. Rocha (Eds.), Tratado de geriatria e gerontologia (2a ed., pp.1436-1443). Rio de Janeiro: Guanabara Koogan.

Folstein, M. F., Folstein, S. E., \& Mchugh, P. R. (1975). A pratical method for grading the cognitive state of patient for clinician. Journal of Psychiatric Research, 12, 189-198.

Gazzale, F. K., Lima, M. S., Tavares, B. F., \& Hallal, P. C. (2004). Sintomas depressivos efatores associados em população idosa no Sul do Brasil. Revista de Saúde Pública, 38 (3), 365-371.

Gatz, M., \& Smyer, M. A. (2001). Mental health and aging at the outset of the twenty-first century In J. E. Birren \& K. W. Schaie (Eds.), Handbook of the psychology of aging $\left(5^{\text {th }}\right.$ ed., pp.523-538). San Diego, CA: Academic Press.

Harlow, R. E., \& Cantor, N. (1996). Still participating after all these years: a study of life task participation in later life. Journal of Personality and Social Psichology, 71 (6), 12351249.

Hultsch, D. F., Hertzog, C., Small, B. J., McDonald-Miszczak, L., \& Dixon, R. A. (1992). Short-term longitudinal change in cognitive performance in later life. Psychology and Aging, 7 (4), 571-584.

Hultsch, D. F., Hertzog, C., Small, B. J., \& Dixon, R. A. (1999). Use it or lose it: engaged lifestyle as a buffer of cogntive decline in aging? Psychology and Aging, 14 (2), 245-263.

Irigaray, T. Q., \& Schneider, R. H. (2008). Impacto na qualidade de vida e no estado depressivo de idosas participantes de uma universidade da terceira idade. Estudos de Psicologia (Campinas), 25 (4), 517-525.

Lopes. A. (2006). Trabalho voluntário e envelhecimento: um estudo comparativo entre idosos americanos e brasileiros. Tese de doutorado não-publicada, Universidade Estadual de Campinas.

Lövdén, M., Ghisletta, P., \& Linderberger, U. (2005). Social participantion attenuates decline in perceptual speed in old and very old age. Psychology and Aging, 20 (3), 423-434.

Loures, M. C., \& Gomes, L. (2006). Prevalência de depressão entre alunos da universidade aberta à terceira idade, Universidade Católica de Goiás no início e no término de seu curso. In V. P. Faleiros \& A. M. Loureiro (Eds.), Desafios do envelhecimento: vez, sentido e voz (pp.134-158). Brasília: Universa.

Mackinnon, A., Christensen, H., Hofer, S. M., Korten, A. E, \& Jorm, A. F. (2003). Use it and still lose it? The association between activity and cognitive performance established using latent growth techniques in a community sample. Aging Neuropsychology and Cognition, 10 (3), 215-229.

Maia, L. C., Durante, A. M. G., \& Ramos, L. R. (2004) Prevalência de transtornos mentais em área urbana no norte de Minas Gerais, Brasil. Revista de Saúde Pública, 38 (5), 650-656. 
Mercadante, E. (2002). Comunidade como um novo arranjo social. Revista Kairós, 5 (2), 17-34.

Neri, A. L. (1993). Qualidade de vida no adulto maduro: interpretações teóricas e evidências de pesquisa. In: A. L. Neri (Org.), Qualidade de vida e idade madura (pp.7-55). Campinas: Papirus.

Neri, A. L. (1998) Escala para avaliação de satisfação na vida referenciada a domínios. Manuscrito não-publicado, Universidade Estadual de Campinas.

Neri, A. L. (2003). Qualidade de vida na velhice. In J. R. Rebelatto \& J. R. S. Morelli (Orgs.), Fisioterapia geriátrica (pp.9-34). São Paulo: Manole.

Newson, R. S., \& Kemps, E. B. (2005). General lifestyle activities as a predictor of current cognition and cognitive change in older adults: a cross-sectional and longitudinal examination. Journal of Gerontology: Psychological Sciences, 60B (3), P113-P120.

Petrini, J. K. (1997). Experiência com grupo da 3a idade: um estudo psicológico. Dissertação de mestrado não-publicada, Pontifícia Universidade Católica de Campinas.

Ramos, L. R. (2003). Fatores Determinantes do envelhecimento saudável em idosos residentes em centro urbano: projeto epidoso, São Paulo. Cadernos de Saúde Pública, 19 (3), 793-798.

Rocha, M., Gomes, M. G. C., \& Lima Filho, J. B. (2002). O protagonismo social da pessoa idosa: emancipação e subjetividade no envelhecimento. In E. L. Freitas, L. Py,
A. L. Neri, F. A. X. Cançado, M. L. Gorzoni \& S. M. Rocha (Eds.), Tratado de geriatria e gerontologia (Cap. 123, pp.1030-1036). Rio de Janeiro: Guanabara Koogan.

Salgado, M. A. (1982). Velhice, uma nova questão social (2a ed.). São Paulo: SESC-CETI.

Salthouse, T. A., Berish, D. E., \& Miles, J. D. (2002). The role of cognitive stimulation on the relations between age and cognitive functioning. Psychology and Aging, 14, 483-506.

Scazufca, M., Cerqueira, A. T. A. R., Menezes, P. R., Prince, M., Vallada, H. P., Miyazaki, M. C. O. S., et al. (2002). Investigações epidemiológicas sobre demência nos países em desenvolvimento. Revista de Saúde Pública, 36 (6), 773-8.

Veras, R. P., \& Caldas, C. P. (2004). Promovendo a saúde e a cidadania do idoso: o movimento das universidades da terceira idade. Ciência \& Saúde Coletiva, 9 (2),423-432.

Yesavage, J. A., Brink, T. L., Rose, T. L., Lum, O., Huang, V., Adey, M. B., et al. (1983). Development and validation of a depression screening scale: a preliminary report. Journal of Psychiatric Research, 17 (1), 37-49.

Zelinski, E. M., \& Stewart, S. T. (1998). Individual differences in 16-year memory changes. Psychology of Aging, 13 (4), 622-630.

Recebido em: 19/9/2008

Versão final reapresentada em: 1/10/2009

Aprovado em: 29/1/2010 